\title{
Contribución a la historia de la coqueluche en Chile. Siglo XIX
}

\author{
Enrique Laval $R$.
}

\section{A contribution to the history of pertussis in Chile. XIX Century}

Chilean historians of Medicine do not mention significant pertussis epidemics in the country during XVI, XVII and XVIII centuries, and scarce information is available about epidemics occurred during most of the XIX century, emphasizing one that happened in La Serena in 1851 and Valdivia in 1853. In the last third of the XIX century, epidemics that happened in Santiago as in other Chilean regions were described, reporting that, between 1892 and $1895,8,181$ patients died of pertussis, high numbers most likely due to the severity of epidemics observed since 1890. Measles and pertussis epidemics often co-existed, or were antedated or followed by the other.

Key words: Pertussis, whooping cough, history, epidemics, mortality, measles, coinfection.

Palabras clave: Coqueluche, tos ferina, historia, epidemias, letalidad, sarampión, coinfección.
Pontificia Universidad

Católica De Chile.

Facultad de Medicina

Programa de Estudios

Médicos Humanísticos.

Recibido:20 de junio de 2010 Aceptado: 6 de julio de 2010

\section{Correspondencia a:}

Enrique Laval Román revinf@sochinf.cl

\section{Algunos antecedentes históricos}

Toseph H. Lapin manifestaba en 1943, que "los escritos de los antiguos no mencionaban enfermedad que pudiera parecerse a la tos convulsiva". Seguramente preocupados por las olas epidémicas de influenza y de peste bubónica, la distinción de un tipo poco común de tos debió haberles parecido sin importancia.

La primera mención de la enfermedad se encuentra en El Espejo de la Salud de Moulton, en 1540 y la epidemia más antigua fue descrita por Guillermo de Baillou (1538 1616) en París, el verano de 1578, denominándola "Tussus Quintina". Sin embargo, este acontecimiento sólo fue publicado 62 años más tarde. La epidemia mencionada atacó de preferencia a los niños, ocasionando gran mortalidad.

La enfermedad ha recibido varios nombres, siendo más usados los de tos convulsiva, coqueluche, tos ferina, pertussis y whooping cough (en países de habla inglesa). El de coqueluche, proviene del francés "coqueluchon", que se refiere a la caperuza con que solía guarecerse la cabeza de los enfermos ${ }^{1-3}$.

Sydenham en 1679, describió exactamente la enfermedad y le dio el nombre de pertussis, refiriéndose a una tos violenta de cualquier tipo. Poco después en 1682, Thomas Willis insistió en la peculiaridad epidémica de la que llamó "Tussis Puerorum Seu Suflocativa", diferenciándola de la influenza.

Schnnurrer comunicó una gran epidemia de "Quinta" en 1693, que provocó la muerte de muchos niños en Roma y París. Además de otra en Inglaterra en 1724. Parecería que la enfermedad fue pandémica en Europa en 1732 - 33

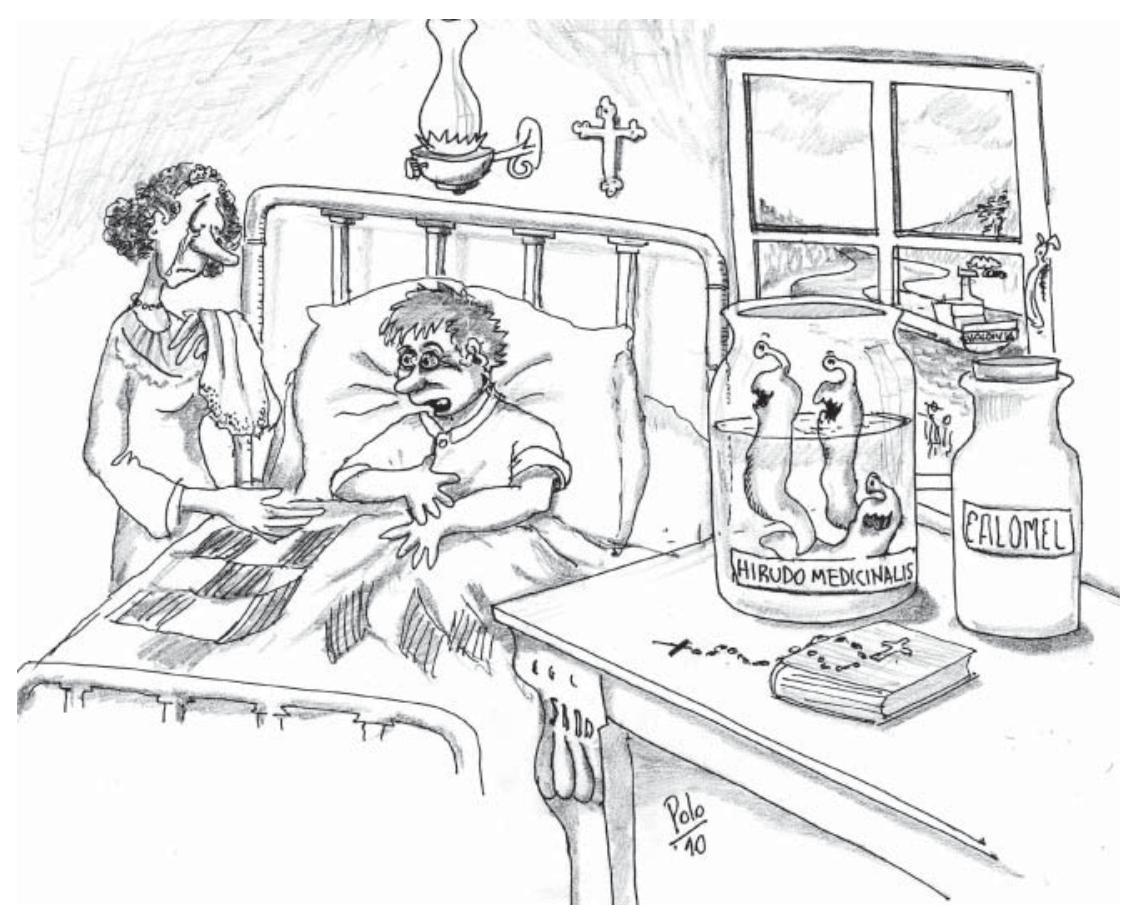

y llevada por marineros ingleses a Jamaica, Perú y Méjico. Entre 1745 y 1748 sucedió una epidemia en Suecia y países vecinos, la que llegó desde África e India. Desde 1832 a 1842 y 1847 a 1849 fallecieron de tos convulsiva 63.381 personas en Inglaterra y Gales ${ }^{1}$.

La coqueluche llegó a ser bien reconocida como una 
entidad clínica en Europa continental a mediados del siglo XVIII. Durante ese siglo y el XIX aumentó su propagación epidémica, adoptando carácter endémico en todos los países civilizados, siendo contadas las regiones donde no existía, por ejemplo Islandia e Islas Faröe ${ }^{1,4}$.

Sería interesante citar lo publicado por Francisco Guerra en su completísima Epidemiología Americana y Filipina 1492-1898, editada por el Ministerio de Sanidad y Consumo de España, en 1999, en relación con epidemias de coqueluche en 1659 y 1730 en algunas regiones de los Estados Unidos de Norteamérica (Nueva Inglaterra y Carolina del Sur, respectivamente) y a fines del siglo XVIII, en todo el territorio de dicho país, así como también en 1865. Según Francisco Guerra las epidemias más importantes en América Latina, en el siglo XIX, comprometieron sobre todo a Costa Rica y Guatemala 5 .

No figura Chile en el escrito de Guerra, tanto en la época colonial como en las siguientes. Nuestros historiadores de la Medicina tampoco refieren epidemias importantes de coqueluche en los siglos XVI, XVII y XVIII, existiendo poca información sobre el siglo XIX.

No obstante, el profesor Wenceslao Díaz en 1874 , consignaba que "en Chile la coqueluche o tos convulsiva sigue los pasos al sarampión; es su hermana gemela, ambas se aúnan o se reemplazan, para empezar en los niños ese sinnúmero de complicaciones cuyo término es muchas veces la muerte. Las epidemias de coqueluche se verifican en todas las estaciones, aunque más ordinariamente a fin de primavera y principios del verano"6.

\section{Epidemia en La Serena en 1851. El Doctor José Juan Bruner}

Veintitrés años antes, José Juan Bruner había dado cuenta de que "en el curso del mes de junio de 1851 estalló en La Serena una epidemia que duró tres meses, que no se limitaba tan solo a la ciudad y provincia de Coquimbo, sino que reinaba en toda la costa occidental de Sudamérica, propagándose de ahí a todo el territorio. El carácter de la epidemia era reumático-catarral, presentándose en forma de una erupción morbilosa (alfombrilla). Al principio se enfermaban niños desde uno hasta diez años, después los adolescentes y luego no escapaban ni los adultos. En otros niños se combinaba con la alfombrilla una tos que gradualmente se volvía convulsiva de catarral que era y su tenacidad ha sido tan grande que aún meses después del completo restablecimiento no quería desaparecer".

Según Bruner la tos convulsiva era de las más violentas que había observado hasta ahora. La periodicidad muy pronunciada de los ataques indicaba su naturaleza nerviosa. "Durante el ataque se levantaba el pulso, latía el corazón, se inyectaba la conjuntiva y la congestión sanguínea se manifestaba de un modo amenazante por la lividez de la cara, por los ojos sobresalientes y en fin por las hemorragias de la boca, nariz y rara vez de los ojos y oídos".

"Puede ser que la habitación perversa de los enfermos de beneficencia en los cuales hice la mayor parte de las observaciones, haya contribuido a la mortandad, pero por otra parte he visto muchos casos bien acomodados"7.

\section{Epidemia en Valdivia en 1853. El doctor Germán Schneider}

El día 30 de noviembre de 1853, el doctor Germán Schneider presentó a la Facultad de Medicina de la Universidad de Chile su Memoria para obtener el grado de Licenciado, revalidando el título de Médico en diciembre de aquel año. Su tesis versó sobre "la enfermedades observadas con mayor frecuencia en Valdivia".

Schneider era alemán, nacido en Magdeburgo en 1820, realizando los estudios de medicina en la Universidad de Bonn. En 1851 fue contratado para prestar sus servicios profesionales en las colonias alemanas instaladas en el sur de Chile, estableciéndose en Valdivia. Refiriéndose a la tos convulsiva señaló "que se mostró epidémica en aquella ciudad, en el mes de mayo de 1852. En los inviernos anteriores, habian acontecido algunos casos esporádicos, pero en 1853 la enfermedad se hizo definitivamente epidémica, extendiéndose poco a poco por toda la provincia". Decía que "fue esta la quinta vez que en mi carrera de médico habia observado la coqueluche en forma epidémica y como antes en Alemania se me han ofrecido las mismas observaciones: 1) las causas del mal han de buscarse en el influjo de la atmósfera, no de la tierra, principalmente estando aquélla repleta de agua suelta y así como en Alemania son importantes los vientos del sur, en Valdivia lo son los del norte; 2) que la enfermedad, sobre todo en el apogeo de la epidemia, está ligada con inflamaciones: bronquitis, laringitis, pleuritis, neumonía, etc; 3) que en párvulos, muchas veces trae consigo una dislocación, principalmente hernia umbilical; 4) que tienen una afinidad muy estrecha los "morbilli" y la tos convulsiva; porque casi siempre los unos suelen seguir a la otra o a la inversa. En la epidemia que tuvimos en Valdivia el año pasado (1852), habiendo cesado la tos convulsiva, luego se presentaba el sarampión, pero con carácter muy benigno".

El doctor Schneider nos dejó numerosas páginas destinadas al tratamiento de diversas enfermedades. Creemos interesante transcribir su método para curar la tos convulsiva: llamado al principio de la enfermedad "procuro precaver sus progresos con un fuerte emético. Si hay alza térmica, sinoca (fiebre continua), antiflogosis en toda su extensión, sangrías, sanguijuelas, calomel, nitro, digital. Cuando no hay fiebre prescribo las unciones 
de ungüento de tártaro estibiado en el epigastrio y en el trayecto del pneumogástrico". Prosigue Schneider: "a los apuntes sobre la tos convulsiva agrego algunos sobre el hidrocéfalo agudo, aquella enfermedad maligna, a que muchas veces pasa el anterior, resultando entonces la muerte. Luego, si terminado el paroxismo de la coqueluche suceden convulsiones, se ensanchan las pupilas y entra en coma; entonces el pronóstico es pésimo. En caso de hallarse ya los enfermos en este estado y con parálisis, me he limitado, con el único objeto de tranquilizar a los dolientes, a una asistencia meramente paliativa por no ser posible ya salvar la vida. Concluyendo lo poco que me parecía digno de ser comunicado sobre mi método de curar la tos convulsiva, creo tener motivos para advertir que la regla de que mientras más remedios en cualquiera enfermedad se ponderan como especificos, más incierto suele ser el mismo modo de curarla, lo que puede también aplicarse a esta enfermedad. ¿Qué remedios no han sido recomendados ya! Unciones espirituosas, etéreas y oleosas, el agua fría, tartarus stiliatus y kali carbonium, ferrum y avidum sulphuricum, cociosidilla y china, sanium y avidum benzocium, liquor amonnii, etc, etc" ${ }^{\prime, 9}$.

\section{La coqueluche en los últimos cuarenta años del siglo XIX}

En los últimos cuarenta años del siglo XIX existen algunas otras comunicaciones sobre la tos convulsiva en Chile, como la del doctor José Grossi en 1884, del estudio de las patologías en el valle del Río Maipo, diciendo que las afecciones más comunes son las del tubo digestivo $(26,5 \%)$ e inmediatamente después las enfermedades pulmonares $(16,5 \%)$, señalando para la coqueluche un $4,5 \%$. Relata que a partir de una epidemia de bronquitis, que seguía a los cambios de temperatura; de alguno que otro caso de tos convulsiva en niños menores de 5 años, de neumonía por resfrío después de las bacanales (causa ordinaria aquí), la más común de las afecciones del pulmón es la pleuresía o "puntada", como la llaman los mineros ${ }^{10}$.

Tres años antes, Dávila, Contardo y Barros Ovalle, señalan sobre la "constitución médica reinante" en el país que "las únicas epidemias de enfermedades dignas de mencionar, han sido, como en el año anterior, la viruela, la coqueluche y el sarampión. Conjuntamente con la viruela, en los primeros meses, sustituyéndola completamente a partir de junio o julio de 1880, la coqueluche ha mantenido su forma epidémica durante los dos tercios del año, pero sin tener tampoco una extensión alarmante" $"$.

Ricardo Dávila Boza informa en la Revista Médica de Copiapó, 1881, que "hubo numerosos casos de coqueluche, erisipela de la cara, disentería y diarrea catarral en niños durante la primavera, recrudeciendo la tos convulsiva en los últimos meses del año"12.
En la Revista Médica de Provincias, dando a conocer sucesos de Angol, se comunica que "la coqueluche no perdonó a ningún niño, aunque no hubo complicaciones"13.

En el verano de 1887 apareció la tos convulsiva en la zona de Victoria (provincia de Cautín), lo que motivó preocupación, la que se vio reflejada en la prensa. Así, El Mercurio de Valparaíso del día martes 23 de febrero de 1887 da a conocer que "con caracteres alarmantes se está desarrollando en la ciudad de Victoria la enfermedad de la tos convulsiva, por lo que se teme una posible epidemia en la región. El vecino de ese pueblo Nicanor Leiva, ha perdido a dos de sus hijos"14.

En 1890 se produjo una epidemia de coqueluche en Magallanes y en 1894, una de viruela, "por suerte ambas relativamente benignas" ${ }^{15}$. En este último año, el doctor Moisés Amaral Martínez desempeñándose como médico inspector sanitario municipal en Santiago dio cuenta a la Sociedad Médica "que ha tenido ocasión de visitar la Escuela Pública de Niños $N^{o} 14$, calle Sama $N^{o} 4$, donde ha observado varios niños atacados de tos convulsiva. Algunos de estos enfermos han seguido asistiendo a la Escuela". El doctor Amaral pasó un informe al Consejo Superior de Higiene Pública dando cuenta de las pésimas condiciones higiénicas del local por cuyas salas atraviesa una acequia que está cubierta sólo por tablas siendo además estrecha y poco alumbrada. Es su opinión que se debe cerrar la Escuela y rechazar en absoluto a todo niño enfermo que no esté libre de contagio.

En la calle de la Bandera $N^{\circ} 6$ ha podido comprobar 14 casos, uno de los cuales falleció. Es un local insalubre y que la autoridad debe vigilar ${ }^{16}$.

En los años 1890 y 1895 la mortalidad de niños había aumentado por epidemias de sarampión y coqueluche ${ }^{17}$.

Según lo anotado por el doctor Carlos Sommers, entre 1892 y 1895 fallecieron por coqueluche en todo Chile 8.181 enfermos, de los cuales 4.587 fueron mujeres (56\%) y 3.594 hombres (44\%), más que triplicando la letalidad entre 1893 y 1895 , lo que seguramente pudo deberse a la gravedad de las epidemias que ocurrieron a partir de 1890 y quizás un poco antes como puede leerse en una crónica aparecida en la Revista Médica de Chile de 1888-89: "la coqueluche hace en la actualidad numerosas víctimas en nuestro clima. Noticias que estamos viendo en las publicaciones del día manifiestan que en algunas ciudades los niños atacados se cuentan por centenares y los fallecidos en los últimos meses pueden apreciarse en cifras bastante elevadas.

Casi no existe lugar de la República donde la enfermedad no haya alcanzado. Tal vez sea esta una de las epidemias más extensas que hayamos tenido por acá.

A pesar de la magnitud de la calamidad, la alarma no ha sido de proporciones, vale tan poco la vida de los nacionales que se deja morir en su primera edad a generaciones enteras"18,19. 
EI tratamiento de la coqueluche con esencia de ciprés

\section{Recomendaciones para el traslado de los enfermos}

Sería necesario ahora referirse a un tratamiento que se empleó en la tos convulsiva y cuyo ferviente mentor fue el doctor don José Manuel Bravo Bravo, quien después de hacer un exhaustivo resumen de los medicamentos utilizados en dicha enfermedad y que fueron abandonados por ineficacia, pasa a ocuparse de "un nuevo método de tratamiento y del medicamento que le sirve de base, que no tiene cabida todavía en ninguna de las farmacopeas del mundo y que por el buen resultado que ha dado a todos los que han tenido ocasión de emplearlo, merece un lugar preferente en todas ellas".

Tal es el aceite esencial de ciprés, llamado también "Esencia de Mönckeberg", del nombre de su descubridor.

Fue descubierto en el año 1885, de un modo casual por el doctor J.J. Mönckeberg, presidente del Instituto Sanitario Uruguayo de Montevideo.

Durante una gran epidemia de coqueluche que azotó a Montevideo en los años 1885 y 1886, varios niños enfermos fueron enviados a una quinta en los alrededores de aquella ciudad. Para sus juegos escogieron un terreno ocupado por una plantación de cipreses y el doctor Mönckeberg observó con extrañeza la rapidez con que la enfermedad declinó en todos. Creyendo encontrar el secreto de la rápida mejoría en la respiración de aire cargado de los principios aromáticos del ciprés, hizo destilar esencia de este árbol, la que fue usada por él y otros colegas, con resultados satisfactorios.

En 1887 llegó a Chile, enviada por el descubridor a su sobrino médico chileno Carlos Mönckeberg, "precisamente cuando una epidemia de tos convulsiva se iniciaba en el país".

El doctor Bravo trató como primeros casos tres niños del Fundo Lo Prado y a la madre también atacada, los que sanaron en corto plazo.

Se utilizaba en inhalaciones "poniendo 15 a 20 gotas de esencia en la delantera y parte superior de los vestidos del niño. Repitiendo la operación cada vez que se habia evaporado la esencia. Al mismo tiempo se usó en forma de jarabe, por cucharadita de café varias veces en el día, siendo mejor inmediatamente después de los accesos".

Las conclusiones a las que llegó el doctor José Manuel Bravo fueron las siguientes: 1) La esencia de ciprés es el medicamento más rápido para el tratamiento de la tos convulsiva; 2) No tiene ninguno de los inconvenientes de los medicamentos empleados hasta ahora; 3) Es de muy fácil manejo, aplicación muy simple y no se fastidia en nada a los pequeños enfermos los que lo toleran con mucha facilidad y aún con gusto ${ }^{17,20}$.

Antes de finalizar estos "recuerdos del siglo XIX", daremos a conocer lo publicado por el minucioso y gran epidemiólogo de su época, el doctor Ricardo Dávila Boza, sobre los "medios de trasladar a los enfermitos de tos convulsiva sin molestias para el público: el año pasado (1900) decretó la Alcaldía la prohibición de trasladar en tranvías y en coches de posta a enfermos de tos convulsiva u otras afecciones contagiosas. Pero no es aceptable dejar a los conductores la facultad de rechazar a un pasajero. Por otra parte entre las medidas tomadas contra la tos convulsiva, existe la de hacer respirar a los enfermos aire puro. De ahi la necesidad que ellos tienen de usar alguno de estos medios de transporte, sea para buscar sitios bien ventilados dentro de la ciudad misma, sea para irlos a buscar en otros pueblos y en este caso usan naturalmente los ferrocarriles".

"Un ministro francés ha dictado las siguientes disposiciones dirigidas principalmente a los jefes de estación y que son adaptables a Chile: 1) Todo carro debe ser construido de material desinfectable; deberá ser aseado y desinfectado en la estación de término; 2) Cada carro llevará las escupideras necesarias para el uso de los pasajeros; 3) Los departamentos aislados que tienen algunos carros serán reservados exclusivamente para los enfermos.

Por el momento se puede permitir el acceso de los niños enfermos a la "imperial" de los carros cerrados y a todos los carros abiertos"21.

A comienzo del siglo XX, específicamente en el año 1906, los microbiólogos belgas Bordet y Gengou, aislaron un "bacilo gram negativo pequeño, aeróbico estricto, patógeno exclusivo del ser humano", reconocido como el agente causal de la enfermedad (Bordetella pertussis). Posteriormente se producirían hitos importantes en el diagnóstico clínico y de laboratorio, así como sobre el tratamiento y en la prevención de la coqueluche por la vacunación ${ }^{22,23}$.

\section{Resumen}

Los historiadores chilenos de la Medicina no relatan epidemias importantes de coqueluche en el país durante los siglos XVI, XVII y XVIII, con poca información disponible sobre las sucedidas en casi todo el siglo XIX, destacándose la de La Serena en 1851 y Valdivia en 1853. En el último tercio del siglo XIX, hubo comunicaciones sobre epidemias ocurridas, tanto en Santiago como en otras regiones de Chile, señalándose que entre 1892 y 1895 fallecieron por coqueluche 8.181 enfermos, lo que pudo deberse a la gravedad de las epidemias que se produjeron a partir de 1890. Es interesante indicar que, con bastante frecuencia, co-existieron epidemias de sarampión y coqueluche, o bien estas últimas fueron precedidas o seguidas por aquellas. 


\section{Referencias}

1.- Lapin J H. Whooping cough. Ed. Charles C. Thomas. USA 1943.

2.- Olea M A. Tos ferina (coqueluche). El Vigía. 2003, 7: 37-8.

3.- Pedro-Pons A. Tratado de Patología y Clínica Médicas. Tomo VI. Enfermedades Infecciosas. Salvat Ed. Barcelona. España. 1952.

4.- Brugsh T. Tratado de Patología Médica. Tomo I. Ed. Labor. Barcelona. España. 1937

5.- Guerra F. Epidemiología americana y filipina. 1492-1898. Ministerio de Sanidad y Consumo. Madrid. España. 1999.

6.- Díaz G W. Geografía Médica de Chile. Capítulo III. De las enfermedades epidémicas. Rev Méd Chile 1874; 3: 329-52./ AUCH 1875; 47: 86-130.

7.- Bruner J J. Sobre la epidemia de 1851 en La Serena. AUCH 1854; 11: 39-42.

8.- Schneider G. Memoria presentada ante la Universidad de Chile (Facultad de Medicina). AUCH 1853; 10: 580-8.

9.- Laval M E. Historia del Hospital San Juan de Dios de Santiago. Impta. Stanley. Santiago de Chile. 1949.

10.- Grossi J. Contribución al estudio de la geografía médica de la República de Chile. Rev Méd Chile 1884-85; 13: 27-37.

11.- Dávila B R, Contardo J, Barros Ovalle P N. Constitución médica reinante. Enero de 1881. Rev Méd Chile 1880; 9;
249-455.

12.- Dávila B R. Revista Médica de Copiapó, en 1881. Rev Méd Chile 1881; 10: 454-5.

13.- Autor no mencionado. Revista Médica de Provincias. Angol. Rev Méd Chile 1882; 11: 26-31.

14.- El Mercurio de Valparaíso. 23 de Febrero de 1887.

15.- Martinic B M. La Medicina en Magallanes. Ed. La Prensa Austral. Punta Arenas. Magallanes. Chile. 2009.

16.- Sociedad Médica de Santiago. Sesión de Conferencias del 5 de octubre de 1884. Rev Méd Chile 1894; 22: 486-8.

17.- Vargas N. Historia de la Pediatría Chilena. Crónica de una alegría. Ed. Universitaria. Santiago de Chile. 2002.

18.- Sommers C. La mortalidad en Chile, durante los años 1892, 1893, 1894 y 1895. Rev Ch Hig 1896; 3: 374-95.

19.- Crónica. La coqueluche hace estragos. Rev Méd Chile 1888-89; 17: 525.

20.- Bravo J M. Tratamiento de la tos convulsiva por la esencia de Ciprés. Rev Méd Chile 1890-91; 19: 337-53.

21.- Sesión de conferencias. Sociedad Médica. 18 de octubre de 1901. Rev Méd Chile 1901; 29: 328-30.

22.- Abarca V K, García C P, Vial P. Microbiología Clínica. Ed. Universidad Católica de Chile. Santiago de Chile. 2001.

23.- Repetto D G, Abarca V K, Concha B M, Giaconi G J. Enfermedades Infecciosas Inmunoprevenibles. Ed. Universidad Católica de Chile, Santiago de Chile. 2003. 